\section{E-065 "RESCUE" INTRACRANIAL STENTING FOR REFRACTORY LARGE VESSEL (RE)OCCLUSION IN ACUTE ISCHEMIC STROKE}

G Toth, S John, M Elgabaly, M Hussain, M Bain. Cerebrovascular Center, Cleveland Clinic, Cleveland, $\mathrm{OH}$

\subsection{6/neurintsurg-2016-012589.137}

Introduction The use of intracranial stents for symptomatic cerebrovascular stenosis has been limited since the publication of recent clinical trials suggestive of elevated procedural risks. However, the utility of these devices for "salvage" therapy in acute stroke has not been well studied.

Objective To investigate the efficacy of intracranial stenting in acute ischemic stroke patients with large vessel occlusion, who failed to recanalize or continued to reocclude with standard thrombectomy methods.

Methods Retrospective data review and analysis. Data was collected in 2015-2016.

Results We identified 5 patients (3 males; mean age 45.2 years), who received intracranial stents during acute stroke intervention. Stents were utilized if vessel reocclusion occurred after thrombectomy (4 cases), or when recanalization could not be achieved with standard thrombectomy devices (1 case). Self-expanding stents were used in 3, and balloon-mounted stents were deployed in 2 cases. Intraprocedural abciximab, followed by postprocedural dual antiplatelet therapy was used in all cases to prevent in-stent thrombosis. Total procedure time was 116-162 min (mean $134.6 \pm 19.8$ ). First recanalization was achieved within 22-106 min (mean $47.8 \pm 34.1$ ). Final TICI $2 \mathrm{~B}$ or 3 recanalization was achieved in all 5 (100\%) patients. Clinical follow-up ranged from 4 days to 8 months. No symptomatic intracranial hemorrhage occurred. Median preprocedure vs follow-up NIHSS were 19 vs 11, respectively. One patient with the longest recanalization time (106 min), and highest preprocedural NIHSS (27) died. Another patient with reocclusion a few hours after a previous thrombectomy procedure did not improve despite TICI 3 final recanalization. The other 3 patients (60\%) showed clinical improvement: the median NIHSS has decreased from 14 to 7 . Conclusion Intracranial stent placement for refractory large vessel (re)occlusion in acute ischemic stroke is feasible with high technical success rate, however, this is not always associated with favorable clinical outcome. If refractory vessel occlusion or reocclusion is encountered during acute stroke intervention, early stent placement and recanalization may lead to more favorable outcome. Verification in a larger cohort is necessary.

Disclosures G. Toth: None. S. John: None. M. Elgabaly: None. M. Hussain: None. M. Bain: None.

\section{E-066 ENDOVASCULAR TREATMENT OF MCA ANEURYSMS - A SINGLE CENTER CASE SERIES}

R Padmanabhan, S Power. Neuroradiology, James Cook University Hospital, Middlesbrough, UK

\subsection{6/neurintsurg-2016-012589.138}

Introduction The ideal treatment modality for both ruptured and unruptured MCA aneurysms is a contentious subject. Both clipping and endovascular therapy (EVT) of these aneurysms are viable options. So far there have been no randomized controlled trials that have compared the results of clipping versus endovascular therapy for MCA aneurysms.
We present a consecutive series of 30 MCA aneurysms (ruptured and unruptured) that were treated using endovascular techniques in our center between Jan 2015-Feb 2016. The use of novel endovascular aneurysm treatment techniques and adjunctive devices is discussed. We also describe the procedural complications and the immediate and short-medium term results of their clinical and imaging follow up.

Materials and methods A retrospective analysis of the PACS database at James Cook University Hospital identified 30 episodes of "embolization of MCA aneurysms" performed in 29 patients between 1 Jan 2015-29 Feb 2016.

The relevant admission case notes, angiography and crosssectional imaging and follow- up clinic data were reviewed. Results 30 MCA aneurysms in 29 patients (24 Female, 5 male) with an age range from 26-79 years were treated using EVT during this period. There were 14 ruptured and 16 unruptured MCA aneurysms. 10 of the 14 ruptured MCA aneurysms had associated parenchymal haematomata.

The median size of aneurysm treated was $5 \mathrm{~mm}$ (range 1.3 $\mathrm{mm}-13 \mathrm{~mm})$. Adjunctive devices were used in 21 patients comprising of balloons in 15 cases, low profile stents in 4 cases, 1 web device and 1 PCONUS device. Novel endovascular techniques were used in 8 cases which included dual balloon remodeling technique in one case, dual micro-catheter in 4 cases, balloon with dual microcatheters in 2 cases and neck remodeling with guidewire support in one case.

There were no intra, peri-procedural or delayed ruptures, coil prolapse/migration in either of the ruptured or unruptured MCA aneurysms groups.

Intra-procedural clot formation was noted in 3/29 (10.3\%) patients which was treated with IV absciximab with no sequalae. $1 / 29(3.4 \%)$ patients had a groin haematoma post stent assisted embolization secondary to failure of groin closure device.

$15 / 16$ patients in the unruptured cohort had no change in their $\mathrm{mRs}$ score post procedure at discharge. $\mathrm{mRs}$ score of $1 / 16$ patients with groin haematoma who needed surgical evacuation, returned to the pre-operative baseline at clinic review in 3 months.

$5 / 14$ patients with aneurysmal SAH died as a result of their original hemorrhage. The remaining 9 returned to a $\mathrm{mRs}$ score of $0-2$ at clinic review.

Follow up imaging at 1 year is available in $10 / 29$ patients, small neck remnants are noted in 5/10 cases. Two of the remnants have developed in patients with aneurysmal SAH with haematoma at presentation. In the other 3 cases deliberate neck remnants were left at the time of original treatment which have not changed.

Conclusion Endovascular treatment of MCA aneurysms is efficacious and has a good safety profile with acceptable immediate and mid-term results. Continues innovation in endovascular techniques and devices has enabled safe and effective embolization of both ruptured and unruptured MCA aneurysms.

Disclosures R. Padmanabhan: None. S. Power: None.

\section{E-067 ENDOVASCULAR APPROACH IN THE REPAIR OF THE MIDDLE CEREBRAL ARTERY ANEURYSM INCLUDING THOSE WITH WIDE NECK AND COMPLEX IN MORPHOLOGY-A CASE SERIES}

Y Lodi, V Reddy. Neurology, Neurosurgery and Radiology, Upstate Medical University/UHSWilson Medical Center, Johnson City, NY

10.1136/neurintsurg-2016-012589.139 